\title{
La question des nations dans les villes médiévales tchèques comme problème méthodologique
}

Jan Vojtíšek

\section{(2) OpenEdition}

1 Journals

Édition électronique

URL : http://journals.openedition.org/ifha/8051

DOI : 10.4000/ifha.8051

ISSN : 2198-8943

Éditeur

IFRA - Institut franco-allemand (sciences historiques et sociales)

\section{Édition imprimée}

Date de publication : 31 décembre 2014

ISSN : 2190-0078

\section{Référence électronique}

Jan Vojtíšek, "La question des nations dans les villes médiévales tchèques comme problème

méthodologique », Revue de l'IFHA [En ligne], 6 | 2014, mis en ligne le 31 décembre 2014, consulté le 15 novembre 2019. URL : http://journals.openedition.org/ifha/8051 ; DOI : 10.4000/ifha.8051

Ce document a été généré automatiquement le 15 novembre 2019.

(CIFHA 


\title{
La question des nations dans les villes médiévales tchèques comme problème méthodologique
}

\author{
Jan Vojtíšek
}

1 Le Moyen Âge tchèque est marqué par la cohabitation de deux groupes ethniques, les Tchèques et les Allemands ${ }^{1}$. Ceux-ci sont attestés en Bohême depuis le Xe siècle, présents à la cour princière comme prélats et dans l'entourage du duc. La première chronique importante de Bohême, la chronique de Cosmas (†1125) nous renseigne bien sur la présence des Allemands. Cosmas se penche particulièrement sur la question des clercs étrangers auxquels il s'oppose hardiment ${ }^{2}$. On trouve chez lui une remarque frappante concernant l'élection d'un nouvel évêque de Prague : le clergé a crié sa joie en latin, le peuple en tchèque et l'entourage princier dans une langue qui était assimilée à de l'allemand ${ }^{3}$, traduisant le fait que la cour préférait l'usage de l'allemand ${ }^{4}$. N'oublions pas que les épouses des ducs de Bohême - et donc les mères de futurs ducs étaient pour la plupart des princesses de l'Empire ${ }^{5}$. On rencontre aussi dans les sources des mentions de la présence de marchands étrangers (des Allemands, des Juifs et des Wallons) et on possède une charte des lois pour les Allemands en Bohême, c'est-à-dire pour ces marchands étrangers datant des années $1170^{6}$.

2 Les XIIe et XIIIe siècles constituent le moment décisif pour la formation de la structure ethnique et démographique de la Bohême médiévale. Ce phénomène s'inscrit dans le contexte d'essor agricole, démographique et économique de l'ensemble de l'Occident chrétien. En Bohême, on assiste à un accroissement démographique intérieur mais aussi à une immigration considérable venue des régions voisines surpeuplées ${ }^{7}$. Le développement démographique n'était cependant pas seul en cause. On peut noter au cours du XIIIe siècle tout un ensemble de changements économiques et sociaux ${ }^{8}$. Il faut souligner la naissance de véritables villes « institutionnellement » fondées sur les droits municipaux. L'institution et la culture municipales proviennent en Bohême du milieu allemand. On distingue les circuits du droit municipal liés aux villes de l'Empire9. Parallèlement au fait que les noms de la plupart des bourgeois, bien que rares dans les 
sources de Bohême, sont d'origine allemande ${ }^{10}$, l'historiographie considérait traditionnellement les villes médiévales du royaume de Bohême comme une véritable « citadelle allemande » dans les pays tchèques, jusqu'à la révolution hussite.

3 Le problème des nations en Bohême ne peut pas être étudié en faisant abstraction de son historiographie. Cette question a joui d'une attention considérable depuis la naissance de l'historiographie tchèque au sens moderne au XIXe siècle. Animé par l'esprit de « renaissance nationale » tchèque, le débat s'est montré particulièrement vif sur ce point et les historiens patriotiques se sont heurtés à leurs homologues d'origine allemande. La question des nationalités médiévales a été politisée surtout aux moments où se décidait le destin des nations dans la Monarchie autrichienne et où se recomposait l'arrangement politique en Europe centrale ${ }^{11}$. La recherche est donc devenue un politicum. La discussion s'est rallumée à l'occasion de la question " allemande » dans la République tchécoslovaque, atteignant un point culminant avec la montée en puissance d'Adolf Hitler. Une théorie dite "anticolonisationnelle " apparaît dans ce contexte. Elle prétend que les peuples germaniques établis en Bohême dans les premiers siècles de notre ère n'étaient jamais partis vers la Bavière, mais s'étaient seulement réfugiés dans les montagnes frontalières, d'où ils sont redescendus au XIIIe siècle pour s'installer au centre du pays. De ce droit des premiers habitants, ces historiens prétendaient faire découler le droit des Allemands sur la Bohême ${ }^{12}$. La plupart des études sur les nations dans les villes médiévales tchèques se situent dans ce contexte de l'instrumentalisation de l'histoire en vue de l'argumentation nationale, voire nationaliste. C'est surtout la position importante des Allemands dans les villes d'une part et les sources privilégiées pour l'étude de la composition ethnique que forment les livres municipaux de l'autre qui ont attiré l'attention des chercheurs sur le milieu urbain.

4 Les livres municipaux apparaissent en Bohême au début du XIVe siècle comme une étape du développement de l'administration municipale. Ils prennent modèle sur les productions comparables élaborées dans l'Empire, surtout en Saxe. Dans un premier temps, un seul livre a servi à noter toutes sortes d'affaires, tant publiques que privées. On y trouve mélangés les privilèges municipaux, les arbitrages, les achats et ventes, les dettes, les contrats de mariage ou les testaments. C'est le cas des livres appelés " manuels du conseil municipal ». Plus tard, émergera une spécialisation des livres (sous la forme par exemples des livres des testaments), mais l'évolution s'observe qu'au XVe siècle ${ }^{13}$.

Comme ces sources énumèrent les habitants des villes, la méthode de l'analyse onomastique, croisée avec l'approche statistique s'est naturellement imposée aux chercheurs. La méthode d'enquête consiste dès lors à diviser les noms en trois catégories: les noms d'origine slave (c'est-à-dire tchèque), les noms d'origine germanique (c'est-à-dire allemands) et les noms neutres, qui sont en majorité des noms bibliques. Tels furent les résultats statistiques livrés sur la proportion des Tchèques et Allemands. Une telle approche a pourtant suscité bien des interrogations et provoqué un vif débat parmi les historiens ${ }^{14}$.

6 Tout d'abord, il faut constater que les livres municipaux ne sont pas une source uniforme. Chacun d'entre eux, du fait de sa typologie particulière, a ses limites et ses avantages. Ainsi, il est toujours nécessaire d'interroger la représentativité des notices inscrites par rapport à l'ensemble de la société urbaine. Le cas des « manuels du conseil municipal » présente un bon exemple. La quasi-totalité thématique des insertions laisse 
croire à une valeur générale de la source. Pourtant, on sait que seules les couches aisées, principalement les élites urbaines, sont mentionnées dans le corpus, et donc la source témoigne uniquement pour ce groupe d'habitants ${ }^{15}$. Ensuite, les registres de l'impôt municipal ou d'autres comptes, qui semblent parfaitement désignés pour une étude statistique, ne contenant quasiment que des noms et des chiffres, posent d'autres problèmes pour leur exploitation. Pour la ville de Brno, on possède plusieurs registres de l'impôt municipal du XIVe siècle ${ }^{16}$. Leur comparaison soulève l'un des problèmes essentiels de la méthode onomastique : la forme non codifiée des noms médiévaux. La même personne est parfois inscrite sous plusieurs noms dans les registres différents. Par exemple, en 1343 apparaît un tonnelier nommé « Wernushus doleator ", qui d'après le suffixe pourrait être identifié comme un Tchèque. En 1345, nous rencontrons un «Wernlinus pinter » dont le nom suggère la nationalité allemande. Néanmoins, il s'agit de la même personne ${ }^{17}$.

7 Cela nous amène à la question de la langue employée par le scribe. La langue des sources étant généralement le latin, c'est le scribe qui influence la forme des noms. Ce sont surtout les noms personnels et locaux qui révèlent la langue d'origine du scribe. Il peut avoir une tendance à préférer sa langue en transcrivant les noms ou surnoms des gens et leurs métiers, et à déformer parfois les noms de l'autre langue. Un principe, formulé par Bedřich Mendl dans les années 1930, s'impose alors pour la recherche : si l'on trouve le nom en forme tchèque dans la source écrite par un scribe de langue allemande, il est permis de supposer que la personne était effectivement Tchèque. Mais si l'on trouve dans la même source le nom en forme allemande, cela ne doit pas signifier nécessairement que cette personne est Allemande, et vice vers $a^{18}$. L'ensemble de la théorie s'applique naturellement aux indications des noms aussi que des métiers.

Mais cette méthode est bien sûr liée à un autre problème. Le nom n'indique pas nécessairement l'appartenance ethnique ou nationale de la personne en question. Ce sont surtout les familles patriciennes allemandes, dont l'origine ne fait aucun doute, qui nous le prouvent. Dans la famille du maître de monnaie Eberhard au milieu du XIIIe siècle, l'un des fils était appelé Jaroš, forme diminutive du nom typiquement slave Jaroslav $^{19}$. Le nom Venceslaus pose le même problème. Ce nom tchèque apparait en quantité non négligeable dans les familles patriciennes allemandes. On ne peut pas considérer Venceslas comme un nom tchèque, mais plutôt comme un nom neutre. Une seule situation permet, avec précautions, de conclure à la nationalité de la personne : si son nom est enregistré sous la forme tchèque Václav chez un scribe allemand. On trouve cette problématique également pour les noms allemands, tel Ulrich et son diminutif tchèque Uluš, ou bien dans sa version tchèque oldřich ${ }^{20}$. L'historiographie explique ces phénomènes par l'influence des mères dans les familles mixtes, ou par celle des parrains. Chez les patriciens, on explique les noms tchèques par les aspirations de ceux-ci à s'élever dans la noblesse tchèque ${ }^{21}$. Cependant, il ne faut pas négliger des interactions et des influences culturelles entre deux groupes ethniques qui se côtoient depuis longtemps. La transmission des noms fait clairement partie de ces échanges et de ces transmissions, accélérés par la cohabitation dans un même pays. Ils peuvent même s'effectuer en l'espace d'une génération comme le montre bien la situation de Danelaw en Angleterre ${ }^{22}$. Cette culture hybride saxo-viking possède des similarités notables avec la réalité de la Bohême médiévale. La "mode " joue aussi un rôle non négligeable et il faut la considérer comme un facteur dans l'analyse. 
9 Dans les recherches onomastiques sur les nationalités dans les villes médiévales, on a laissé de côté une question pourtant tout à fait essentielle, à savoir la définition de la nation dans les villes médiévales tchèques. Les historiens ont procédé en fonction de leur perception de la nation. Mais sa définition pose évidemment des problèmes. On en comprend plus ou moins le sens, mais on a du mal à le cerner comme une catégorie précise. J'exposerais pour ma part quatre marques distinctives par lesquelles on construit son identité en se définissant par rapport aux autres : l'origine ethnique, la langue, la culture et l'appartenance juridique (c'est-à-dire politique ${ }^{23}$. Dans la communication avec les autres (dans un sens large), on utilise ou on accentue une ou plusieurs catégories selon leur valeur distinctive et selon l'objectif. Or à lire, les sources médiévales de Bohême, on trouve exactement la même stratégie. L'analyse du sentiment national et de son développement dans le royaume de Bohême a été conduite par František Graus pour l'époque pré-hussite et par František Šmahel pour l'époque hussite $^{24}$. Les résultats montrent que les critères étaient les mêmes au Moyen Âge, et que chaque auteur médiéval accentue ceux qui sont les plus importants pour lui et son propos. Par exemple, la chronique dite de Dalimil du début du XIVe siècle met l'accent principal sur la langue ${ }^{25}$. Pendant les affrontements nationaux à l'Université de Prague au début du XVe siècle, les universitaires ont mis l'accent sur la langue, mais aussi très fortement sur les origines ethniques ${ }^{26}$.

10 L'autre trait important tient à l'apparente variabilité des sentiments nationaux dans les sources. Le sentiment national comporte deux niveaux. Le niveau de base est formé par la conscience latente de l'appartenance à un groupe et par la distinction par rapport à un autre. Elle est toujours présente et passive. Le second niveau est l'expression d'un sentiment national actif, toujours lié à un objectif concret. Celui-ci tend de façon générale à une meilleure préservation des moyens de vivre d'un groupe ou d'un seul initiateur, donc à un pouvoir politique et économique. L'expression active de la nation est alors un fait politique. À côté de la défense contre un danger extérieur, l'expression active de la nationalité représente l'un des moyens de restreindre la participation aux avantages économiques ou politiques afin de préserver pour soi la position la plus favorisée. C'est ce que montrent pour les villes tchèques, les sources témoignant de restrictions nationales, pourtant très rares, tels les statuts des corps de métiers limitant l'accès à une nation ${ }^{27}$. Ces sources signalent donc non seulement une certaine évolution démographique, mais surtout des changements économico-politiques dans la ville.

11 La majeure partie des sources municipales (c'est-à-dire les livres municipaux) ne livre pas, à cause de leur fonction administrative les informations explicites sur le sentiment national dans les villes. C'est l'une des raisons pour lesquelles la méthode fondée sur l'étude des noms a été utilisée dans le passé pour répondre à des impératifs liés à la politique du temps. J'ai montré dans la présente contribution l'aspect problématique de cette approche et les risques liés à des résultats imprécis. Je ne refuse cependant pas cette méthode entièrement car elle conserve à mes yeux une certaine utilité malgré ses défauts. Mais il faut la combiner avec d'autres pistes d'interrogation, comme l'a fait de façon remarquable Jaroslav Mezník, surtout pour la Vieille-Ville de Prague, mais aussi pour la Nouvelle-Ville, en croisant les noms, les métiers et la socio-topographie urbaine $^{28}$. Du point de vue démographique, les études sur l'immigration dans les villes (les nouveaux venus, les nouveaux contribuables de l'impôt urbain) ou sur les origines régionales des habitants pourraient vérifier et nuancer les théories de la bohémisation des villes démographiquement passives par l'arrivée des gens des villages voisins, et 
peut-être expliquer le caractère allemand de longue date de certaines villes ${ }^{29}$. De même, on a constaté que les modèles exogènes pouvaient jouer un rôle important dans le choix du nom. Il parait donc profitable de mener une étude sur la popularité des noms et sur son développement. À côté de résultats sans doute intéressants, elle pourrait fournir une nouvelle perspective pour l'étude de la division nationale des noms.

Ensuite, il faut se tourner vers des témoignages particuliers du sentiment national enregistrés dans les livres municipaux. Par exemple, dans la ville de Kolín, on trouve dans les livres du dernier quart du XIVe siècle quelques bourgeois identifiés par le surnom Bohemus ${ }^{30}$. On peut interroger la signification de cette indication à la valeur distinctive incontestable. Il est difficile de penser que la ville de Kolín ne comptait pas une certaine proportion de Tchèques avant la fin du XIVe siècle. On doit donc chercher l'explication ailleurs. Bohemus pourrait être un nom de famille hérité d'une période où la grande majorité des habitants de Kolín étaient des Allemands, ou bien il pourrait s'agir d'un nom désignant des Tchèques appartenant à un groupe ou à un métier qui étaient encore dans les mains des Allemands.

Toute une série de questions se pose par rapport à la vie dans un milieu bilingue. Tout d'abord, comment s'effectuait l'administration de la ville et quels étaient les lieux centraux de la vie sociale par rapport aux langues? Quelle langue était utilisée dans l'espace public et quelle était la politique à l'égard des langues? Quelle était la division linguistique dans la sphère religieuse ou dans les métiers? Dans les sources, on constate qu'il existe des prédicateurs spécialisés, allemands et tchèques. Peut-on distinguer de façon générale certains métiers monopolisés par un groupe national, comme on le voit à Prague? Un autre champ pour lequel l'étude des livres municipaux se montre particulièrement féconde, repose sur l'étude des relations familiales, ou du moins celles des familles des élites et des couches aisées de la société urbaine. L'établissement de ces liens permettrait aussi de nuancer et vérifier la méthode statistique appliquée aux noms. Bref, toutes les recherches proposées se dirigent vers une analyse des rapports quotidiens des deux nations et du fonctionnement de leur cohabitation dans un espace limité. Seule l'analyse en profondeur des livres municipaux sur la base de méthodes sociotopographiques, prosopographiques et microhistoriques pourrait approfondir nos connaissances sur ces aspects nationaux de la vie urbaine médiévale.

14 Nous avons aussi abordé la question importante des tensions nationales qui se traduisent par des conflits du pouvoir. Dans les sources littéraires, et dans quelques sources diplomatiques isolées, on peut constater des tensions nationales dans les villes tchèques au cours du XIVe siècle et avant la « révolution » hussite. Naturellement, les livres municipaux n'en témoignent pas explicitement, mais la reconstruction des listes des membres du conseil et leur étude prosopographique détaillée, plaçant l'accent sur les liens familiaux et sur leurs clientèles, c'est-à-dire sur les liens économiques et sociaux, peut permettre d'esquisser la structure de la "société politique » de la ville, et ainsi apporter des réponses à la problématique des nations. 


\section{NOTES}

1. Cet article a été mené à bien grâce au soutien de l'Agence tchèque de la recherche (GA ČR) dans le cadre du projet P405/12/G148 "Les codes culturels et leurs transformations à l'époque hussite ".

2. Voir le dialogue du duc Břetislav II. de Bohême et marquis Wiprecht de Groitche, Cosmae Pragensis chronica Boemorum, Bethold Bretholz (éd.), Berlin : Weidmannische Buchhandlung, 1923, III, 7, p. 167-168.

3. Ibid., I, 23, p. 45-46; František Graus, «Die Bildung eines Nationalbewusstseins im mittelalterlichen Böhmen. Die vorhussitische Zeit », Historica, n 13, 1966, p. 19.

4. La remarque de Cosmas nécessiterait une analyse approfondie.

5. Sur les génealogies de la maison des Přemyslides, Dušan Třeštík/Josef Žemlička/Petr Sommer (dir.), Přemyslovci. Zrození státu, Praha : Nakladatelství Lidové noviny, 2010, p. 548-573.

6. Codex juris municipalis regni Bohemiae I., Jaromír Čelakovský (éd.), Praha : Edvard Grégr, 1886 , n. 1, p. 1-3. Sur son interprétation voir Jiří Kejřr, «Zwei Studien über die Anfänge der Städteverfassung in den böhmischen Ländern ", Historica, n 16, 1969, p. 81-142.

7. Josef Vítěslav Šimák, České dějiny I/5. Středověká kolonisace v zemích českých, Praha : Jan Laichert, 1938 ; Jiří Sláma, « Příspěvek k vnitřní kolonisaci raně středověkých Čech », Archeologické rozhledy, n 19, 1967, p. 433-445.

8. Josef Žemlička, Počátky Čech královských 1198-1253. Proměna státu a společnosti, Praha: Nakladatelství Lidové noviny, 2002; Jan Klápště, Proměna českých zemí ve středověku, Praha: Nakladatelství Lidové noviny, 2010.

9. Jiří Kejř, Vznik městského zř́zení v Čechách, Praha : Karolinum, 1998.

10. Passim dans les éditions des sources diplomatiques aussi que narratives de l'époque préhussite.

11. František Kutnar/Jaroslav Marek, Stručné dějiny českého a slovenského dějepisectví, Praha: Nakladatelství Lidové noviny, 2009.

12. Sur ces débats nationalistes voir Josef Žemlička, Počátky Čech královských 1198-1253, op. cit., p. 11-21.

13. Sur les livres municipaux voir Václav Vojtíšek, O studiu městských knih českých, Praha : Václav Vojtíšek, 1916; Ludmila Sulitková, Vývoj městských knih v Brně ve středověku (v kontextu vývoje městských knih v českých zemích), Praha/Brno : Archiv Akademie věd ČR/Archiv města Brna, 2004.

14. Cette méthode sert de la base pour la plupart des études spécialisées. Une présentation est faite par František Šmahel, « Výsledky a výhledy výzkumu národnostní skladby českých měst od konce 13. do počátku 15. století » in : Richard Marsina (dir.), Národnostný vývoj miest na Slovensku do roku 1918, Martin : Osveta, 1984, p. 239-254.

15. Martin Nodl, «Středověký testament jako abnormalita » in : Kateřina Jíšová/Eva Doležalová (dir.), Pozdně středověké testamenty $v$ českých městech. Prameny, metodologie a formy využití, Praha: Scriptorium, 2006, p. 73-85.

16. Knihy počtů města Brna z let 1343-1365, Bedřich Mendl (éd.), Brno : Československý státní ústav historický, 1935.

17. Bedřich Mendl, «O národnostním složení středověkého Brna », Časopis Matice moravské, $n^{\circ}$ 62, 1938, p. 365.

18. Ibid., p. 366.

19. Jan Vojtíšek, «Mincmistr Eberhard a jeho potomci. Působení jedné gründerské rodiny ve středověkých Čechách », in : Martin Musílek (dir.), Havelské Město pražské ve středověku. Historie archeologie - stavební historie, Praha : Cassablanca, 2012, p. 158-183.

20. Bedřich Mendl, « O národnostním složení středověkého Brna », art. cit., p. 366. 
21. Ibid., p. 367.

22. Wendy M. Hoofnagle/Wolfram R. Keller (dir.), Other Nations. The Hybridization of Medieval Insular Mythology and Identity, Heidelberg : Winter, 2011.

23. Je m'appuie au départ sur les remarques de Shannon Lewis-Simpson, "Assimilation or Hybridization? A Study of Personal Names from Danelaw » in : Wendy M. Hoofnagle/Wolfram R. Keller (dir.), Other Nation, op. cit., p. 15-19.

24. Ces deux œuvres forment toujours une base d'études de nations dans les pays tchèques au Moyen Âge, František Graus, «Die Bildung eines Nationalbewusstseins", art. cit., p. 5-49; František Šmahel, Idea národa v husitských Čehách, Praha : Argo, 2000².

25. Staročeská kronika tak řečeného Dalimila. Vydání textu a veškerého textového materiálu I-II, Jiř́i Daňhelka et alii (éd.), Praha : Academia, 1988.

26. František Šmahel, Idea národa v husitských Čehách ; op. cit., p. 34-49.

27. František Šmahel, «Lingua et sanguis: pokrevní společenství 'jazykového národa' ve středověkých Čechách », Časopis Matice moravské, n 117, 1998, p 407-416.

28. Jaroslav Mezník, « Národnostní složení předhusitské Prahy », Sborník historický, n 17, 1970, p. 5-30.

29. Martin Nodl, «Národnostní vývoj předhusitského Stř́ibra. (Modelová prosopografická analýza) », Český časopis historický, n 94, 1996, p. 528-559.

30. Liber contractuum I et Liber contractuum II (1376-1401), Státní okresní archiv Kolín (sans signature).

\section{AUTEUR}

\section{JAN VOJTÍŠEK}

(Université Charles de Prague/Université Paris-Sorbonne) 